\title{
Topics in torsion theory
}

\author{
Stelios Charalambides
}

The purpose of this thesis is to generalize to the torsion-theoretic setting various concepts and results from the theory of rings and modules. In order to accomplish this we begin with some preliminaries which introduce the main ideas used in torsion theory, the major ones being $\tau$-torsion and $\tau$-torsionfree modules as well as $\tau$-dense and $\tau$-pure submodules. In the first chapter we also introduce a new concept, that of a $\tau$-compact module, which is basic enough to deserve a place among the preliminaries.

The results that we obtain fall into three areas which are to a certain degree interrelated. The first area is on $\tau$-Max modules, which we introduce as a torsion-theoretic analogue of Max modules. The main aim is to generalize a well-known result by Shock which characterizes Noetherian rings by using the socle, the radical and Max modules. All of these concepts have torsion-theoretic counterparts which we utilize in our generalization. Furthermore, we define and characterize left $\tau$-Max rings and apply the torsion-theoretic version of Shock's theorem to obtain a characterization of $\tau$-short modules motivated by a recent article in which short modules were introduced.

The second area deals with various flavours of $\tau$-injectivity, some known and some new. We introduce $\tau$ - $\mathcal{M}$-injective and s- $\tau-\mathcal{M}$-injective modules and examine their relationship with the known concepts of $\tau$-injective and $\tau$-quasi-injective modules. We then provide an improved version of the Generalized Fuchs Criterion which characterizes s- $\tau-\mathcal{M}$-injective modules, and give a generalization of Azumaya's Lemma. We also prove that every $M$-generated module has a $\tau$ - $\mathcal{M}$-injective hull which is unique up to isomorphism and show how this is linked to the $\tau$-quasi-injective hull. We then examine $\Sigma-\tau$-injectivity, generalizing well-known results by Faith, Albu and Năstăsescu and Cailleau which provide necessary and sufficient conditions for the $\Sigma$-s- $\tau$-injective property, the $\Sigma$-s- $\tau$ - $\mathcal{M}$-injective property and for a direct sum of $\Sigma$-s- $\tau-\mathcal{M}$-injective modules to be $\Sigma$-s- $\tau$ - $\mathcal{M}$-injective.

In the third area we introduce some new concepts with the aim of bringing to the torsion-theoretic setting the concept of a CS or extending module. The approach is twofold. The first is via $\tau$-CS modules which serve as a generalization of CS modules

Thesis submitted to the University of Otago, July 2006. Degree approved, October 2006. Supervisor: Dr John Clark.

Copyright Clearance Centre, Inc. Serial-fee code: 0004-9727/07 $\$ A 2.00+0.00$. 
as well as $\tau$-quasi-continuous, $\tau$-quasi-injective and $\tau$-injective modules, and the second is via s- $\tau$-CS modules which are a special case of CS modules. Our motivation is to provide a torsion-theoretic analogue of a well-known result by Okado which characterizes Noetherian modules. We have some partial results using s- $\tau$-CS modules and a nice torsion-theoretic analogue, albeit without the use of $\tau$-CS or s- $\tau$-CS modules. We also examine the relationship between our relative versions of CS modules with those of other authors and obtain refinements to some of their results.

Department of Mathematics and Statistics

University of Otago

Dunedin, PO Box 56

New Zealand

e-mail: stelios@maths.otago.ac.nz 